\title{
The HPOL Spectropolarimeter at Ritter Observatory
}

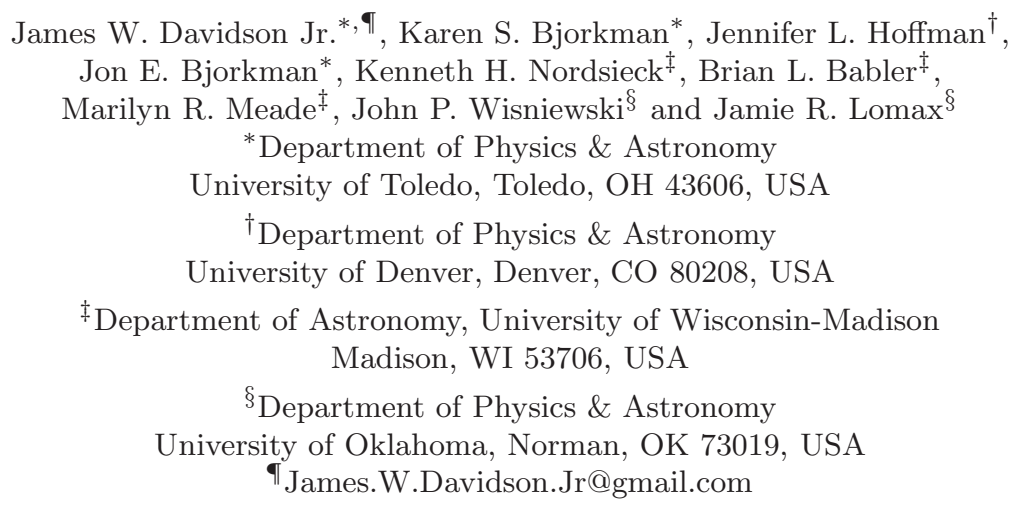

Received 2014 June 26; Revised 2014 October 15; Accepted 2014 November 16; Published 2014 December 23

The HPOL spectropolarimeter, previously operated by the University of Wisconsin at Pine Bluff Observatory (PBO), has been restored and relocated to the University of Toledo's Ritter Observatory. We achieved first light with HPOL at Ritter Observatory on 2012 March 11. We present a detailed instrument description of HPOL in its current configuration at Ritter Observatory, and a description of the standard observing procedure. We performed frequent observations of unpolarized standard stars to calibrate the instrumental polarization. We present polarimetric calibration results for observations spanning 2012 March 11 to 2013 May 2. Our calibration work shows that the systematic uncertainties of observations obtained with HPOL at Ritter Observatory are comparable to, and sometimes outperform, the systematic uncertainties obtained at PBO. We have successfully recommissioned the HPOL spectropolarimeter at Ritter Observatory, making it once again available for ongoing science observations.

Keywords: Instrumentation: polarimeters, polarization, techniques: polarimetric.

\section{Introduction}

The University of Wisconsin's (UW) Half-wave Spectropolarimeter (HPOL) was installed as a dedicated instrument at the 36-inch telescope at Pine Bluff Observatory (PBO) located outside of Madison, WI, in 1989 January (Wolff et al., 1996; Nordsieck \& Harris, 1996). HPOL was originally a ground-based support instrument for the Wisconsin Ultraviolet Photo-Polarimeter Experiment (WUPPE), one of the telescopes flown as part of NASA's Astro package aboard the Space Shuttle in 1990 (STS-35, Astro-1) and 1995 (STS67, Astro-2) (Bjorkman et al., 1993; Nordsieck et al., 1994). HPOL also has been mounted to the WIYN $3.5 \mathrm{~m}$ telescope on several observing runs to Kitt Peak National Observatory outside of Tucson, Arizona. Over the years at PBO, HPOL underwent a number of upgrades and modifications, the most significant being a changing from a Reticon dual channel photo-diode array to a Reticon $400 \times 1200 \mathrm{CCD}$ in 1995 . Numerous spectropolarimetric studies have been conducted with HPOL over many years, including topics such as: classical Be stars, B[e] stars, pre-main sequence stars, luminous red variable stars, interacting binaries, Wolf-Rayet stars, the interstellar

"Current address: Department of Physics, Albion College, Albion, MI 49224, USA. 
medium, novae and supernovae. ${ }^{(a)}$ A subset of HPOL data from PBO observations is available to the community via the NASA MAST website hosted by the Space Telescope Science Institute. ${ }^{(b)}$

In 2004, two separate equipment failures sidelined HPOL at PBO. The first was a failure of the slit camera, used for target acquisition. The second and more serious issue was a failure of the hard drive in the control computer. The instrument remained out of service until around 2007, when our group began efforts to restore HPOL to operation. We restored and reconfigured the control system software and installed a new slit camera. We then relocated HPOL to the University of Toledo's Ritter Observatory 1-meter telescope, where it achieved first light on 2012 March 11 (Davidson, 2013).

In this paper, we start with a description of the HPOL instrument in its current configuration at Ritter Observatory in Sec. 2. In Sec. 3, we discuss the observing procedure. In Sec. 4, we discuss our instrumental polarization calibration work and compare the results to previous calibration work for HPOL at PBO. Finally, in Sec. 5 we summarize HPOL's performance at Ritter Observatory.

\section{Description of the Instrument}

The HPOL spectropolarimeter is an extensively modified model 26767 Boller \& Chivens spectrograph, designed to measure linear polarization as a function of wavelength. The parameters used to describe partial polarization are known as Stokes parameters after first being defined by Stokes (1852), and then rediscovered by Chandrasekhar (1950). For a chronology of astronomical polarization see Tinbergen (1996) and Gehrels (1974). For additional background information on polarization see Clarke (2010). There are four Stokes parameters: $I, Q, U$ and $V$. Stokes $I$ is the total intensity, $Q$ and $U$ are used to describe the linear polarization, and $V$ describes the rotation of the polarization vector for circular and elliptical polarization. However, HPOL was designed with a halfwave plate (Sec. 2.5 below) and no quarter-wave plate; it thus measures linear polarization $(I, Q, U)$ but not circular polarization $(V)$.

The wavelength coverage of HPOL is 3200 to $10,500 \AA$ in two grating positions, discussed in

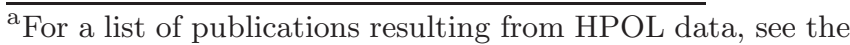
instrument web page at http://www.sal.wisc.edu/HPOL/.

${ }^{\mathrm{b}}$ http://archive.stsci.edu.

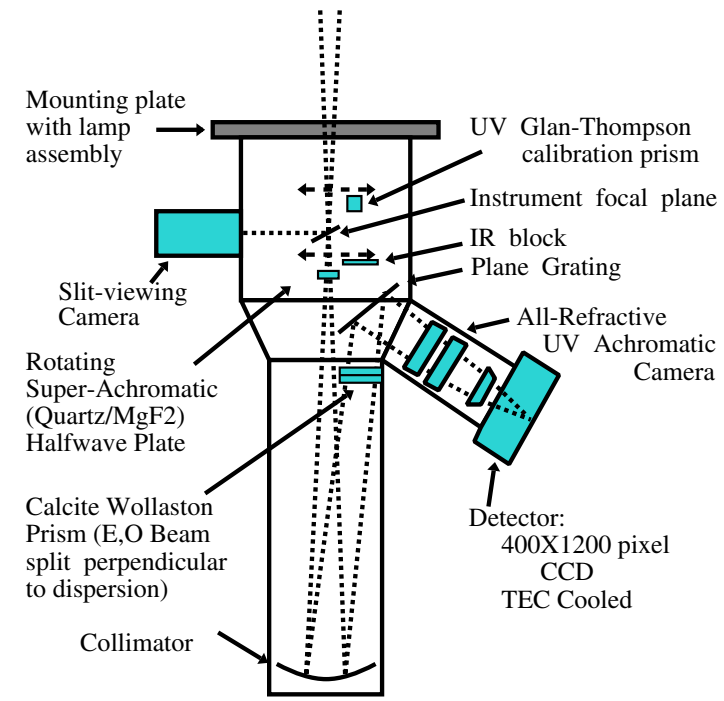

Fig. 1. The HPOL spectropolarimeter optical design.

Sec. 2.8, with a resolution of $7.5 \AA$ below $6000 \AA$ and $10 \AA$ above $6000 \AA$. $^{(c)}$ Figure 1 shows a schematic layout of HPOL with most of the components labeled. Previous discussions of HPOL have been presented by Wolff et al. (1996), Nook (1990) and Nordsieck \& Harris (1996). However, combined, these sources do not provide a complete description of HPOL in its current configuration, particularly with the CCD detector and with the new location at Ritter Observatory. Below we discuss the individual components of HPOL in order of the optical path, from telescope to detector. The Ritter Observatory 1-meter telescope is a Ritchey-Chrétien design with an $\mathrm{f} / 8$ beam. However, an alternative secondary mirror can be used to create an $\mathrm{f} / 13.5$ beam, which is used for HPOL. In this alternate configuration the telescope is of a hybrid design, since both the primary and secondary mirrors are hyperboloids and the entire field of view is not coma free.

\subsection{Arc and flat lamp assembly}

Mounted to the underside of the instrument mounting plate is a slide assembly which houses the arc and flat field lamps, used for wavelength and detector calibration, respectively. This assembly is remotely activated via the instrument control software and moved into the optical path by a small stepper motor. A potentiometer measures the positioning of this assembly to ensure the lamps are

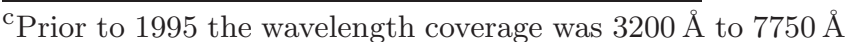
with a resolution of $25 \AA$ (Wolff et al., 1996).
} 
within a specified range before they can be turned on. A tungsten lamp is used for flat fielding while a $\mathrm{HgAr}$ Oriel $\mathrm{P} / \mathrm{N}: 6035$ pencil style calibration lamp, often referred to as a 'Penray' lamp, is used for wavelength calibration. When not in use, the assembly is moved out of the optical path such that all parts are outside the entrance aperture of the instrument.

\subsection{Polarizing prism}

The first optical component inside HPOL is a UV Glan-Thompson calibration prism. This prism can be manually inserted into the optical path to produce $100 \%$ polarized light across the entire wavelength coverage of the instrument. Observations of bright stars with the prism allowed Nordsieck \& Harris (1996) to characterize the wavelength dependence of the half-wave plate position angle. The prism is otherwise outside the optical path for normal observations.

\subsection{Slit and decker assembly}

At the focal plane of this instrument sits a slit and decker assembly consisting of two surface aluminized Dow Fotoform glass plates. Figure 2 depicts the five possible slit sizes and eight possible decker options. Most observations at Ritter are carried out with slit \#3 and decker \#5; this combination creates two apertures, each with a size of $\sim 5.4^{\prime \prime} \times$ $10.8^{\prime \prime}$. On the underside of the slit and decker mounting bracket is a Uniblitz VS25 shutter that is connected to the Photometrics control box for the detector. This shutter takes $3.0 \mathrm{~ms}$ to open and
$5.0 \mathrm{~ms}$ to close, and features a $25 \mathrm{~mm}$ aperture, large enough for all slit and decker combinations.

\subsection{Filters}

Below the slit and decker assembly is a filter slide which houses three filters: red, clear and IRblocking. The filters are used in combination with the two gratings, described in Sec. 2.8, to eliminate diffracted or scattered light. Observations with the red grating use the red filter while observations with the blue grating use the clear filter. For targets which may have large amounts of IR flux, the IR blocker can instead be used with the blue grating to suppress overlap from the second-order spectrum.

\subsection{Half-wave plate}

HPOL uses a rotating super-achromatic halfwave plate consisting of four alternating plates of magnesium fluoride and crystal quartz that was manufactured by Karl Lambrecht Corporation. The half-wave plate introduces a phase delay of half a wavelength, or $180^{\circ}$, to the component of the electric field perpendicular to the optical axis of the half-wave plate. It is important that the wave plate be super-achromatic to minimize the wavelength dependence of birefringence, ensuring minimal deviation from a half-wavelength phase delay across the observable wavelengths of the instrument. The wavelength dependence has been characterized and corrected for as discussed in Nordsieck \& Harris (1996). This is crucial to obtaining quality measurements across the entire optical wavelength range, which is one of the advantageous features of HPOL.
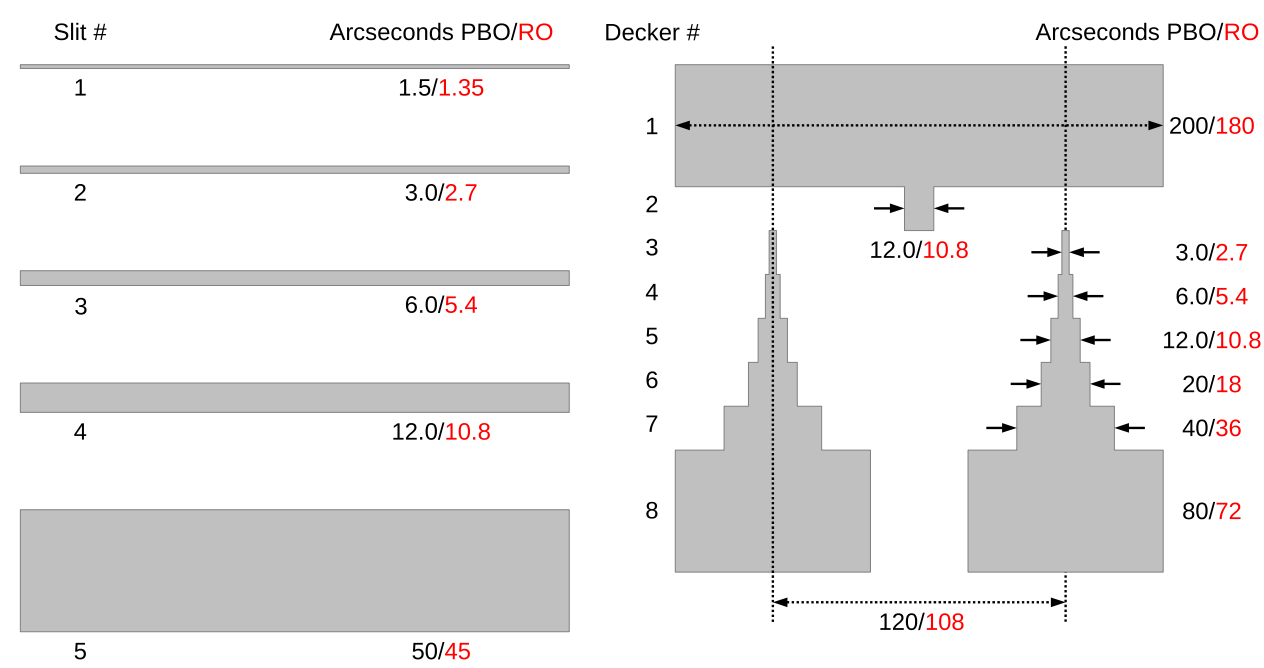

Fig. 2. (Color online) The slit (left) and decker (right) assemblies for HPOL with their corresponding numbers and sizes in arcseconds labeled. Sizes in arcseconds for Ritter Observatory are shown in red. 
A stepper motor and rack-and-pinion drive rotates the waveplate with stops every $11.25^{\circ}$. A solenoid-driven pin fits into a notch on the wave plate assembly at each stop position to lock the wave plate in place. To ensure good repeatability in the positioning of the wave plate, the drive motor is left on to preload the gear. A potentiometer is used to measure the positioning, which is monitored via the control software. This provides measured positioning repeatability to $0.01^{\circ}$. The use of a rotating half-wave plate enables the required observations used to measure the linear polarization of an object, while keeping the instrument orientation fixed with respect to the telescope.

\subsection{Cylindrical lens}

A cylindrical lens mounted to a slide is inserted via control software, oriented such that the magnification is orthogonal to the dispersion direction. This widens the spectrum out over more pixels of the CCD, improving flat field removal and allowing for longer exposure time without saturating the detector. This also allows for binning the readout of the detector which improves readout time. This lens is used for most observations, including all those presented in this paper.

\subsection{Wollaston prism}

After the cylindrical lens and the spectrograph's collimating mirror, the next element is a Wollaston prism constructed from two pieces of calcite crystal, manufactured by Karl Lambrecht Corporation. Originally the prism had optical grease between the elements; however, UW instrument staff removed this grease due to a leak issue, so that now there is only an air gap between the two pieces of calcite. The clear aperture of the Wollaston prism is 52 by $62 \mathrm{~mm}$, and the prism is $10 \mathrm{~mm}$ thick. The prism angle is $3.7^{\circ}$ with an air gap in the middle. Two orthogonally polarized beams (ordinary and extraordinary) are produced, separated by $1.52^{\circ}$ at $3000 \AA$ and $1.22^{\circ}$ at $9000 \AA$, perpendicular to the dispersion of the gratings.

\subsection{Gratings}

HPOL uses two gratings, mounted to a slide arm, that may be selected via the instrument control software. The blue grating is used for the wavelength range of 3000-6200 $\AA$, and the red grating is used for $6000-10,500 \AA$. Both gratings were manufactured by the Milton Roy Company. The blue grating is catalog number 35-53-09-260, and is $68.6 \mathrm{~mm}$ square and $9.1 \mathrm{~mm}$ thick. It has 600 grooves $/ \mathrm{mm}$ with a blaze wavelength of $500 \mathrm{~nm}$ and blaze angle of $8.6^{\circ}$. The factory measured efficiency is $82 \%$ at $480 \mathrm{~nm}, 80 \%$ at $500 \mathrm{~nm}$ and $79 \%$ at $520 \mathrm{~nm}$. The red grating is catalog number 35-53-09580 , and is also $68.6 \mathrm{~mm}$ square and $9.1 \mathrm{~mm}$ thick. It has 400 grooves $/ \mathrm{mm}$ with a blaze wavelength of $8453 \AA$ and blaze angle of $9.44^{\circ}$. The factorymeasured efficiency is $88 \%$ at $8350 \AA, 90 \%$ at $8450 \AA$ and $88 \%$ at $8550 \AA$.

\subsection{Camera}

HPOL covers the entire optical wavelength range, using both gratings, from the UV cutoff at $3000 \AA$ to the near-IR at $10,500 \AA$. The camera used to focus the light onto the detector is a custom-built ultraviolet achromatic camera designed to minimize chromatic aberrations across the entire wavelength range of the instrument. The camera was designed by KHN using all-refractive elements to ensure there are no vignetting effects, which is critical for polarization work. It was installed on 1989 September 28.

\subsection{Detector}

The current detector is a $400 \times 1200$ pixel Reticon CCD. A field flattener has been incorporated into the dewar window to keep the spectrum in focus. The CCD has a Thermoelectric Cooling (TEC) system to chill the detector down to around $-50^{\circ} \mathrm{C}$. A closed-loop chilling system removes the heat produced by the TEC. This system runs a 50/50 mix of distilled water and ethylene glycol through a PolyScience KR-60A flow-through chiller which maintains the mixture around $-20^{\circ} \mathrm{C}$.

\section{Observational Procedure}

The observations presented here, like most observations with HPOL, were obtained using slit \#3 and decker \#5. At Ritter Observatory, this creates two $\sim 5.4^{\prime \prime} \times 10.8^{\prime \prime}$ apertures separated by $180^{\prime \prime}$, with the standard orientation being East/West on the sky. For reference, we refer to these as aperture 0 (to the East) and aperture 1 (to the West). The observing procedure for a target is kept in a database on the HPOL control computer; targets which have not been observed previously require the observer to create a new data entry in the 
Table 1. Observing sequence.

\begin{tabular}{lllll}
\hline & \multicolumn{2}{c}{ Aperture } & & $\begin{array}{c}\text { Instrumental } \\
\text { polarization } \\
\text { Exposure set }\end{array}$ \\
\cline { 2 - 4 } & 0 & 1 & \multicolumn{1}{c}{ Waveplate positions } & components \\
\hline 1,2 & Star & Sky & {$\left[0^{\circ}, 45^{\circ}\right]$} & $Q$ \\
$3,4,5,6$ & Sky & Star & {$\left[0^{\circ}, 45^{\circ}\right],\left[0^{\circ}, 45^{\circ}\right]$} & $Q, Q$ \\
$7,8,9,10$ & Star & Sky & {$\left[0^{\circ}, 45^{\circ}\right],\left[22.5^{\circ}, 67.5^{\circ}\right]$} & $Q, U$ \\
$11,12,13,14$ & Sky & Star & {$\left[22.5^{\circ}, 67.5^{\circ}\right],\left[22.5^{\circ}, 67.5^{\circ}\right]$} & $U, U$ \\
$15,16,17,18$ & Star & Sky & {$\left[22.5^{\circ}, 67.5^{\circ}\right],\left[11.25^{\circ}, 56.25^{\circ}\right]$} & $U, Q+U$ \\
$19,20,21,22$ & Sky & Star & {$\left[11.25^{\circ}, 56.25^{\circ}\right],\left[11.25^{\circ}, 56.25^{\circ}\right]$} & $Q+U, Q+U$ \\
$23,24,25,26$ & Star & Sky & {$\left[11.25^{\circ}, 56.25^{\circ}\right],\left[33.75^{\circ}, 78.75^{\circ}\right]$} & $Q+U, Q-U$ \\
$27,28,29,30$ & Sky & Star & {$\left[33.75^{\circ}, 78.75^{\circ}\right],\left[33.75^{\circ}, 78.75^{\circ}\right]$} & $Q-U, Q-U$ \\
31,32 & Star & Sky & {$\left[33.75^{\circ}, 78.75^{\circ}\right]$} & $Q-U$ \\
\hline
\end{tabular}

associated files. Once the target has been acquired, and is centered in aperture 0 , the observer then executes the observation program on the control computer. The basic observation sequence is shown in Table 1. With the star located in aperture 0 , and aperture 1 containing sky, the starting position of the waveplate for the first exposure, or set of exposures, is $0^{\circ}$. The control software automatically moves the waveplate to the second position of $45^{\circ}$ and takes another exposure(s). The program pauses and waits for the observer to move the telescope to place the star in aperture 1, and thus sky in aperture 0. Again, the program starts an exposure(s) at a waveplate position of $0^{\circ}$, then $45^{\circ}$, then $0^{\circ}$ and finally $45^{\circ}$. This continues, following the sequence order shown in Table 1. Exposure times and number of exposures required are determined by the software based on the magnitude of the star and the instrument configuration. Each waveplate position pair produces a measurement of a single polarization component; e.g. $0^{\circ}$ and $45^{\circ}$ measures, respectively, $Q$ and $-Q$. In this way, the waveplate position pairs in Table 1, indicated by brackets, measure $Q, U, Q+U$ and $Q-U$ for a source while allowing us to simultaneously aquire a measure of the sky background for the other aperture. For completeness we note that we determine the "sky" contribution to a given stellar measurement from the background measured in the same aperture, not during the same exposure. Once all exposures have been taken for a given grating, the observer then executes the calibration routine which moves in the arc and flat lamp assembly (Sec. 2.1) and begins a sequence of flat field and wavelength calibration exposures. After this, another target can be acquired or the sequence may be repeated for the other grating to obtain full wavelength coverage.

Figure 3 shows several raw images taken with HPOL at Ritter Observatory. These observations were made with the red grating in the standard HPOL configuration: slit \#3, decker \#5, cylindrical lens and red filter. The top image shows the spectrum of the star $\gamma$ Boo when it is located in aperture 0 and the middle image shows the spectrum when the same star is in aperture 1 . In both cases the bright spectra are the two polarization components of the stellar spectrum, while the much fainter sky component spectra from the opposite aperture are too faint to be seen. The bottom image is a raw observation of the

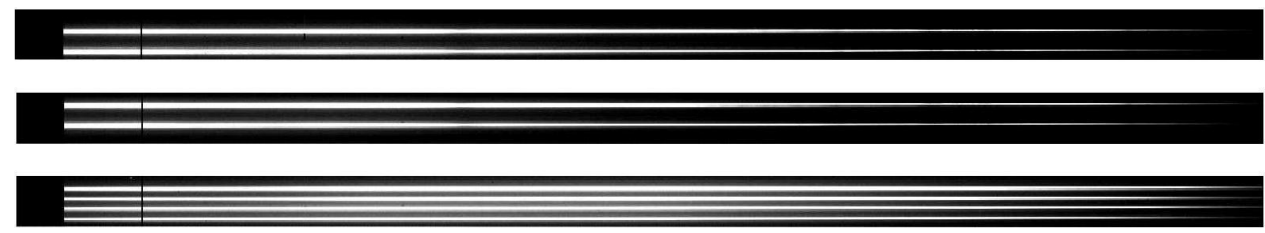

Fig. 3. Raw image examples from HPOL for the star $\gamma$ Boo taken on 2012 April 12 at Ritter Observatory. Top: Image when star is in aperture 0 (and sky in aperture 1). Middle: Image when star is in aperture 1 (and sky in aperture 0). Bottom: Image of a flat field showing all four spectra, ordinary and extraordinary from both apertures. 
flat lamp showing all four spectra, including an ordinary and extraordinary component from each aperture. The two aperture spectra are interlaced vertically in an image (i.e. from top to bottom the spectra are: aperture 1 extraordinary, aperture 0 extraordinary, aperture 1 ordinary, aperture 0 ordinary).

\section{Polarization Calibration}

Observations of star and sky from the two apertures allows for removal of polarization contributions from the sky, most importantly due to scattered light in the atmosphere originating from sources on the ground; these can be significant at Ritter Observatory, which is located on a university campus in a major metropolitan area. Systematic polarization signals from both the instrument and the telescope must also be removed. It is therefore critical to observe unpolarized standard stars, discussed further in Sec. 4.1, in order to determine the systematic polarization for HPOL on the Ritter Observatory 1-meter telescope. Any measured polarization when observing these stars will be due solely to the instrument and telescope since the stars themselves have no measurable intrinsic and interstellar polarization components. While the contributions to the systematic polarization can be determined separately for the telescope and instrument, doing this requires many additional observations. Because the orientation of our system remains fixed, we can easily measure the systematic polarization arising from the instrument and telescope combination. Over time the systematic polarization will also change, for example, as more dust collects on the primary mirror of the telescope, or after the mirrors are cleaned or aluminized. We therefore conduct frequent observations of unpolarized stars to investigate and correct for any possible changes in the systematic polarization with time.

\subsection{Observing unpolarized stars}

Unpolarized standard stars (hereafter "unpols") are typically nearby stars with negligible interstellar polarization. Most unpols are near-solar type stars, as late-type and early-type stars tend to show intrinsic polarization effects. The integrated disk of the Sun, for example, has been measured by Kemp et al. (1987) to have a linear polarization of less than $10^{-7}$ in $\mathrm{V}$ band. Numerous studies have measured the polarization of nearby stars, such as the work of Behr (1959), Serkowski (1974), Piirola (1977), Krautter (1980) and Leroy (1993); the latter includes a catalog of polarization measurements for 1000 stars within 50 pc of the Sun. Long-term monitoring of such systems is critical to investigate potential time variability of such stars.

We have used five unpols listed in Table 2 to calibrate HPOL at Ritter Observatory. We chose these stars because they were previously used as unpols for HPOL during its tenure at $\mathrm{PBO}$, and over that time have not shown any signs of variability or measurable intrinsic polarization. Observations with other instruments support the use of these targets as unpols (see Table 3 for supporting references).

\subsection{Constructing calibrations}

The data reduction and calibration work presented were performed using the REDUCE software package. This is a custom software package created at the Space Astronomy Laboratory at the UW specifically for HPOL (Nook, 1990). We elected to create four separate calibrations for Ritter Observatory observations between 2012 March 11 and 2013 May 2, based on changes to the systematic

Table 2. Unpolarized standard stars used for HPOL calibration.

\begin{tabular}{cccccl}
\hline Star name & HD number & $\begin{array}{c}\text { RA } \\
(\mathrm{J} 2000)\end{array}$ & $\begin{array}{c}\text { DEC } \\
(\mathrm{J} 2000)\end{array}$ & $\begin{array}{c}\mathrm{M}_{V} \\
(\mathrm{mag} .)\end{array}$ & \multicolumn{1}{l}{ Spectral type } \\
\hline$\theta \mathrm{UMa}$ & 82328 & $09: 32: 51.4$ & $+51: 40: 38.3$ & 3.18 & $\mathrm{~F} 7 \mathrm{~V}$ \\
$\beta \mathrm{UMa}$ & 95418 & $11: 01: 50.5$ & $+56: 22: 56.7$ & 2.35 & A0 E \\
$\gamma$ Boo & 127762 & $14: 32: 04.7$ & $+38: 18: 29.7$ & 3.00 & A7III C \\
$\delta$ Cyg & 186882 & $19: 44: 58.5$ & $+45: 07: 50.9$ & 2.90 & B9.5IV $+\ldots$ C \\
$\zeta$ Peg & 214923 & $22: 41: 27.6$ & $+10: 49: 52.0$ & 3.39 & B8V C \\
\hline
\end{tabular}

Note: Values obtained from the SIMBAD database, operated at CDS, Strasbourg, France 
Table 3. Previous observations of unpolarized standard stars.

\begin{tabular}{ll}
\hline Star name & \multicolumn{1}{c}{ References } \\
\hline$\theta$ UMa & $\begin{array}{l}\text { Wolff } \text { et al., 1996 }{ }^{1} \text {; Manset \& Bastien, 1995; Leroy, 1993; } \\
\text { Tinbergen, 1982; Piirola, 1977; Tinbergen, 1979; Behr, 1959 }\end{array}$ \\
$\beta$ UMa & $\begin{array}{l}\text { Bailey et al., 2010; Wolff et al., 1996 }{ }^{1} \text {; Tinbergen, 1982; } \\
\text { Tinbergen, 1979; Piirola, 1977; Behr, 1959 }\end{array}$ \\
$\gamma$ Boo & $\begin{array}{l}\text { Bailey et al., 2010; Bailey et al., 2008; Hough et al., 2006; } \\
\text { Wolff et al., 1996 }{ }^{1} \text {; Appenzeller, 1966; Behr, 1959 }\end{array}$ \\
$\zeta$ Cyg & Bailey et al., 2010; Behr, 1959 \\
\hline
\end{tabular}

${ }^{1}$ Observations made with HPOL at PBO.

polarization over this time. For each of the four time periods, we used REDUCE to construct separate calibrations for apertures 0 and 1 , each with a Stokes $Q$ and $U$ component, resulting in a total of four components. As in our standard reduction procedures, we also used REDUCE to transform the Stokes $Q$ and $U$ values from the instrumental frame into the conventional equatorial frame. Table 4 summarizes the four calibrations, along with the dates and unpols used for each case.

Figure 4 shows all unpol observations in the four components, Stokes $Q$ and $U$ in apertures 0 and 1, used for the first calibration, covering data taken between 2012 March 11 and 2012 April 27. The larger uncertainties at the blue and red ends are due to a decrease in the signal-to-noise, and were also observed in unpol data from PBO. The large feature below $4500 \AA$, seen predominantly in Stokes $Q$, is not seen in PBO data; we suspect this is due to the mirror coating on the Ritter Observatory telescope. We have begun evaluating the mirror coating and will present the results of this study in a future contribution. The pre-calibrated observations for the three other calibrations show similar levels of uncertainty as seen in Fig. 4; however, the polarization variation with wavelength differs between calibrations.

For each calibration, we combined unpol observations for a given aperture and Stokes parameter using equal weighting. We choose equal weighting instead of error weighting to avoid biasing the results to specific unpols which may be brighter, and therefore systematically produce smaller error results. We then Gaussian smoothed the results, in most cases using a FWHM of 37.5 pixels (300 $\AA$ ). However, in certain cases, particularly in Stokes $Q$, the large features on the ends required a Gaussian smooth with a FWHM of 25 pixels $(200 \AA)$. In these cases, we spliced two smoothed curves together, using the 25-pixel FWHM result at the ends and the 37.5-pixel FHWM result in the middle. Figure 5 shows the average results in black and the smoothed results in red for the first calibration. The curves in Fig. 5 have been inverted from those shown in Fig. 4, such that adding the calibration results to the original observations will result in a zero average. Figure 6 shows the four separate calibrations we created for Stokes $Q$ and $U$ in apertures 0 and 1 , along with their corresponding date ranges.

Table 4. Breakdown of calibrations by unpol observations.

\begin{tabular}{|c|c|c|c|c|c|c|c|c|}
\hline \multirow{2}{*}{$\begin{array}{c}\text { Ritter } \\
\text { calibration }\end{array}$} & \multirow{2}{*}{$\begin{array}{l}\text { Start } \\
\text { date }^{1}\end{array}$} & \multirow{2}{*}{$\begin{array}{c}\text { End } \\
\text { date }^{1}\end{array}$} & \multicolumn{5}{|c|}{ Number of unpol observations } & \multirow{2}{*}{$\begin{array}{c}\text { Total number of } \\
\text { observations }\end{array}$} \\
\hline & & & $\theta \mathrm{UMa}$ & $\beta \mathrm{UMa}$ & $\gamma$ Boo & $\delta$ Cyg & $\zeta$ Peg & \\
\hline 1 & 120311 & 120427 & 2 & 4 & 9 & 1 & 0 & 16 \\
\hline 2 & 120511 & 120518 & 0 & 2 & 3 & 1 & 0 & 6 \\
\hline 3 & 120627 & 121214 & 0 & 0 & 3 & 6 & 1 & 10 \\
\hline 4 & 130426 & 130502 & 1 & 1 & 1 & 0 & 0 & 3 \\
\hline
\end{tabular}

${ }^{1}$ The format for the date is YYMMDD. 

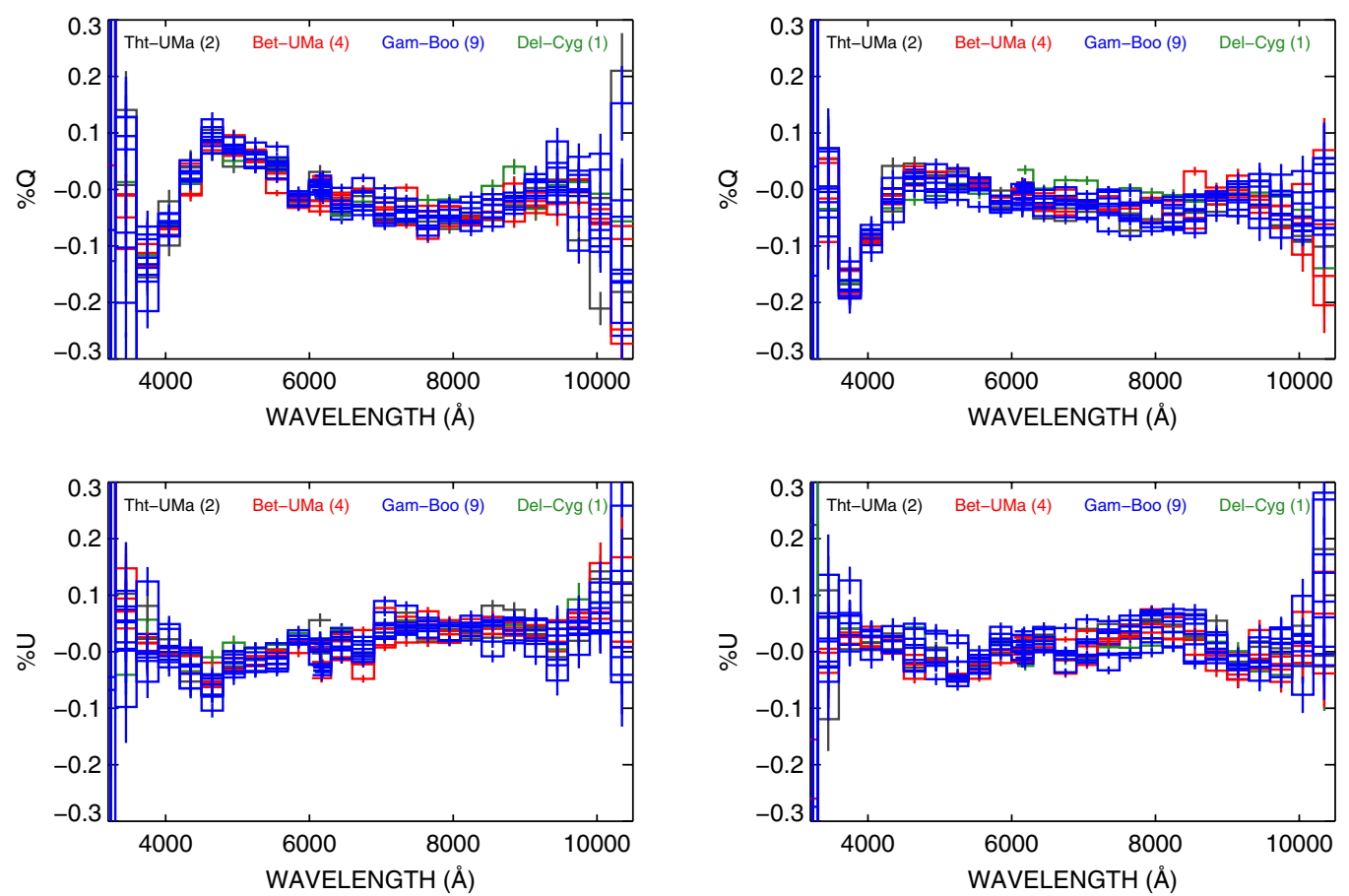

Fig. 4. (Color online) All 16 unpol observations between 2012 March 11 and 2012 April 12 for Stokes $Q$ (top) and Stokes $U$ (bottom) in aperture 0 (left) and aperture 1 (right). Each unpol observation is color coded according to the legend at the top of the plot, which also indicates the number of observations in parentheses. Observations have been binned to $300 \AA$ with corresponding $1-\sigma$ errors.
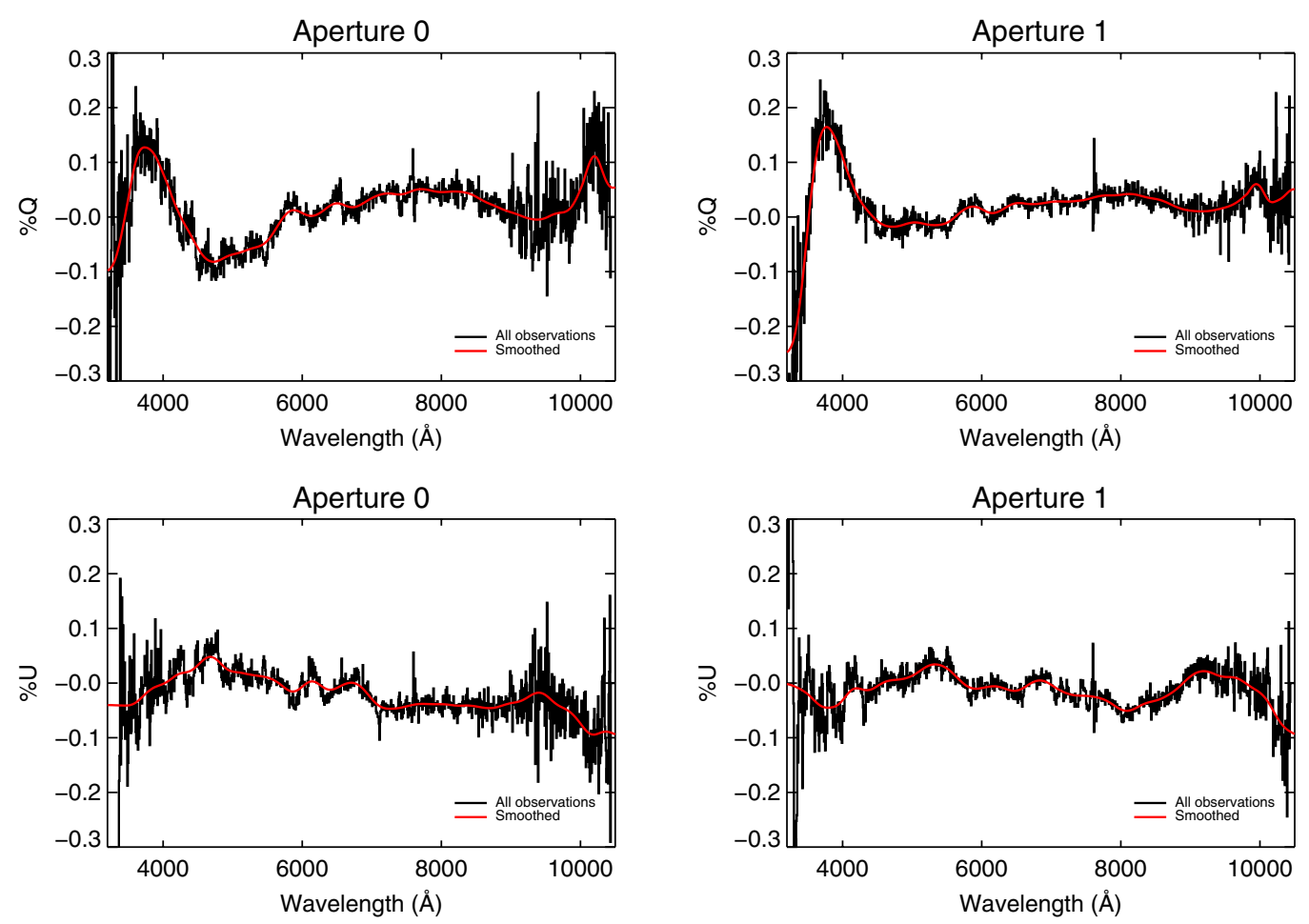

Fig. 5. (Color online) The black lines represent the combination, using equal weighting, of all 16 unpol observations taken between 2012 March 11 and 2012 April 12 for Stokes $Q$ (top) and Stokes $U$ (bottom) in aperture 0 (left) and aperture 1 (right), with no binning. The smoothed red curves are the instrumental polarization spectrum determined by Gaussian smoothing the data in black. 

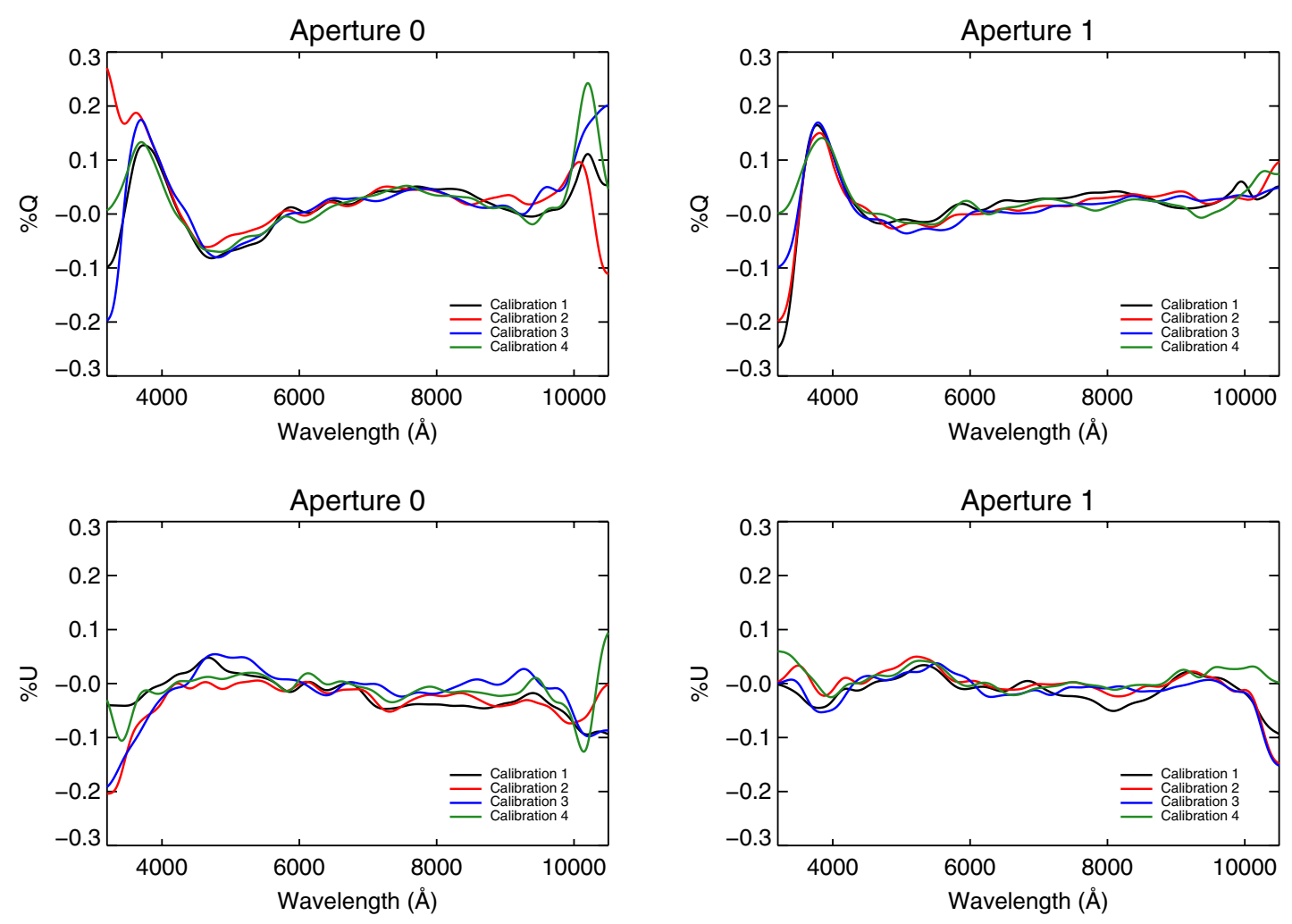

Fig. 6. (Color online) Instrumental polarization curves for Stokes $Q$ (top) and Stokes $U$ (bottom) in aperture 0 (left) and aperture 1 (right) for the time period of 2012 March 11 to 2013 May 2. Effective dates for each calibration are as follows: (1) 2012 March 11 to 2012 April 27; (2) 2012 May 11 to 2012 May 18; (3) 2012 June 27 to 2012 December 14; (4) 2013 April 26 to 2013 May 2.

When we process HPOL data for final release, we use REDUCE to subtract the appropriate instrumental polarization curves from Fig. 6 from the polarized spectra of each target object in each aperture. We then construct the final Stokes $Q$ and $U$ spectra and transform them from the instrumental frame into the conventional equatorial frame.

\subsection{Evaluating the calibrations}

We evaluated each calibration by examining the results when applied to the original observations. We re-reduced all unpolarized observations using the corresponding new calibration file for the date of the observation, following the standard reduction procedures in REDUCE. We then calculated synthetic UBVRI filter polarization data by multiplying the spectropolarimetric data with the normalized Bessell filter transmission curves, as listed in Table 2 of Bessell (1990), and computing a flux weighted integration. For each of the four calibrations, we calculated mean values and associated bias-corrected sample standard deviations for both Stokes $Q$ and $U$ in the $U B V R I$ filters. We take these standard deviations to represent the systematic uncertainties in our filter polarization data, and report them in Table 5 . The values in Table 5 should be taken into account when reporting any filter polarization measurements obtained with the HPOL CCD detector.

\subsection{Comparison with 10 years of previous $P B O$ calibration work}

From 1995 to 2004, observations conducted with HPOL at PBO used the same CCD detector that still remains on the instrument. Thus, we can directly compare the new Ritter observations with the old PBO observations. Figure 7 shows synthetic filter polarization data for all $\mathrm{PBO}$ and Ritter Observatory data used in constructing calibration files. Despite the telescope change, the newer Ritter data are not drastically different. The average spread in a given filter is consistent with $\mathrm{PBO}$ observations or better, as seen in B-band $\% U$, Fig. 7. 
Table 5. Systematic uncertainties from PBO and Ritter Observatory.

\begin{tabular}{|c|c|c|c|c|c|c|c|c|c|c|c|c|c|}
\hline \multirow[b]{2}{*}{$\begin{array}{c}\text { Calibration } \\
\text { number }\end{array}$} & \multirow[b]{2}{*}{$\begin{array}{c}\text { \# of } \\
\text { unpols }\end{array}$} & \multirow[b]{2}{*}{$\begin{array}{l}\text { Start } \\
\text { date }^{1}\end{array}$} & \multirow[b]{2}{*}{$\begin{array}{l}\text { End } \\
\text { date }^{1}\end{array}$} & \multicolumn{2}{|c|}{$U$} & \multicolumn{2}{|c|}{$B$} & \multicolumn{2}{|c|}{$V$} & \multicolumn{2}{|c|}{$R$} & \multicolumn{2}{|c|}{$I$} \\
\hline & & & & $\begin{array}{c}\sigma_{Q} \\
(\%)\end{array}$ & $\begin{array}{c}\sigma_{U} \\
(\%)\end{array}$ & $\begin{array}{c}\sigma_{Q} \\
(\%)\end{array}$ & $\begin{array}{c}\sigma_{U} \\
(\%)\end{array}$ & $\begin{array}{c}\sigma_{Q} \\
(\%)\end{array}$ & $\begin{array}{c}\sigma_{U} \\
(\%)\end{array}$ & $\begin{array}{c}\sigma_{Q} \\
(\%)\end{array}$ & $\begin{array}{c}\sigma_{U} \\
(\%)\end{array}$ & $\begin{array}{c}\sigma_{Q} \\
(\%)\end{array}$ & $\begin{array}{l}\sigma_{U} \\
(\%)\end{array}$ \\
\hline $\mathrm{PBO} 1^{2}$ & 8 & 950124 & 950703 & 0.076 & 0.051 & 0.008 & 0.009 & 0.007 & 0.005 & 0.004 & 0.007 & 0.005 & 0.009 \\
\hline 2 & 8 & 950708 & 960125 & 0.039 & 0.054 & 0.005 & 0.019 & 0.007 & 0.013 & 0.007 & 0.008 & 0.008 & 0.007 \\
\hline $3^{3}$ & 2 & 950703 & 960723 & 0.026 & 0.075 & 0.005 & 0.022 & 0.010 & 0.008 & 0.002 & 0.002 & 0.012 & 0.007 \\
\hline 4 & 4 & 960801 & 961029 & 0.026 & 0.047 & 0.011 & 0.010 & 0.005 & 0.009 & 0.004 & 0.003 & 0.009 & 0.003 \\
\hline $5^{4}$ & $\ldots$ & 970220 & 970429 & $\ldots$ & $\ldots$ & $\ldots$ & $\ldots$ & $\ldots$ & $\ldots$ & $\ldots$ & $\ldots$ & $\ldots$ & $\ldots$ \\
\hline 6 & 5 & 970506 & 970807 & 0.033 & 0.030 & 0.008 & 0.017 & 0.007 & 0.006 & 0.007 & 0.007 & 0.010 & 0.005 \\
\hline 7 & 5 & 970904 & 980113 & 0.040 & 0.074 & 0.006 & 0.028 & 0.004 & 0.012 & 0.008 & 0.006 & 0.008 & 0.007 \\
\hline 8 & 4 & 980321 & 980501 & 0.035 & 0.030 & 0.011 & 0.004 & 0.004 & 0.007 & 0.010 & 0.005 & 0.008 & 0.003 \\
\hline 9 & 3 & 980614 & 980816 & 0.047 & 0.035 & 0.007 & 0.020 & 0.014 & 0.003 & 0.019 & 0.003 & 0.023 & 0.021 \\
\hline 10 & 7 & 981128 & 990811 & 0.014 & 0.035 & 0.017 & 0.016 & 0.010 & 0.005 & 0.010 & 0.010 & 0.011 & 0.009 \\
\hline 11 & 13 & 990904 & 000505 & 0.099 & 0.071 & 0.027 & 0.025 & 0.020 & 0.012 & 0.011 & 0.010 & 0.016 & 0.011 \\
\hline 12 & 12 & 000716 & 010801 & 0.069 & 0.113 & 0.024 & 0.018 & 0.017 & 0.008 & 0.009 & 0.008 & 0.020 & 0.017 \\
\hline 13 & 5 & 011103 & 020228 & 0.052 & 0.054 & 0.017 & 0.020 & 0.009 & 0.008 & 0.004 & 0.006 & 0.010 & 0.004 \\
\hline 14 & 6 & 020515 & 021227 & 0.060 & 0.034 & 0.022 & 0.016 & 0.010 & 0.013 & 0.009 & 0.015 & 0.011 & 0.012 \\
\hline 15 & 19 & 030101 & 041010 & 0.109 & 0.085 & 0.020 & 0.028 & 0.008 & 0.012 & 0.008 & 0.010 & 0.025 & 0.010 \\
\hline Ritter 1 & 16 & 120311 & 120427 & 0.089 & 0.059 & 0.011 & 0.010 & 0.006 & 0.011 & 0.007 & 0.006 & 0.008 & 0.012 \\
\hline 2 & 6 & 120511 & 120518 & 0.077 & 0.076 & 0.014 & 0.005 & 0.009 & 0.008 & 0.007 & 0.002 & 0.009 & 0.016 \\
\hline 3 & 10 & 120627 & 121214 & 0.048 & 0.070 & 0.010 & 0.006 & 0.011 & 0.009 & 0.006 & 0.005 & 0.010 & 0.012 \\
\hline 4 & 3 & 130426 & 130502 & 0.037 & 0.077 & 0.009 & 0.013 & 0.005 & 0.012 & 0.001 & 0.003 & 0.012 & 0.008 \\
\hline
\end{tabular}

${ }^{1}$ The format for the date is YYMMDD.

${ }^{2}$ Previous publications presenting HPOL data may include systematic uncertainties that vary slightly from those presented here; we have recalculated these uncertainties for purposes of this study, but expect differences from past reported values to be negligible.

${ }^{3}$ Because this calibration included data from only two targets, its systematic uncertainties should be treated with caution.

${ }^{4}$ The orientation of HPOL with respect to the PBO telescope varied during this time for the purpose of observing Comet Hale-Bopp. Systematics during this time are not well understood, and thus we have intentionally omitted them from the table.

To quantify the relation between HPOL's performance at PBO and Ritter, we compared the calculated systematic uncertainties in the calibrations at both observatories. Table 5 shows the systematic uncertainties for the HPOL calibrations at Ritter Observatory compared with updated uncertainties for the PBO calibrations, in both cases using data obtained with the CCD detector. The systematic uncertainties at Ritter Observatory are all within the range of previous systematic uncertainties at $\mathrm{PBO}$, as shown in Fig. 8. This comparison reaffirms that in its new location, HPOL is producing data that is comparable in quality to, and in some cases better than, the data it produced at PBO between 1995 and 2004.

\section{Summary}

We have successfully restored the HPOL spectropolarimeter and relocated it to Ritter Observatory, on the University of Toledo campus. We have presented a detailed description of HPOL in its current configuration at Ritter Observatory, as well as provided a summary of the standard observing procedure.

We constructed four separate polarimetric calibrations spanning 2012 March 11 to 2013 May 2. The polarimetric calibration results show that at Ritter Observatory HPOL is performing as well as it was at PBO. HPOL observations of unpolarized standard stars obtained at Ritter show excellent agreement with previous observations obtained at PBO. We also find good agreement between the two observatories in observations of polarization standard stars, which we will present in an upcoming contribution. The similar instrument performance between the two sites shows that polarimetric observations can be obtained at an observatory on a university campus without significantly impacting data quality. Our efforts have resulted in HPOL being once again available for ongoing science observations. 

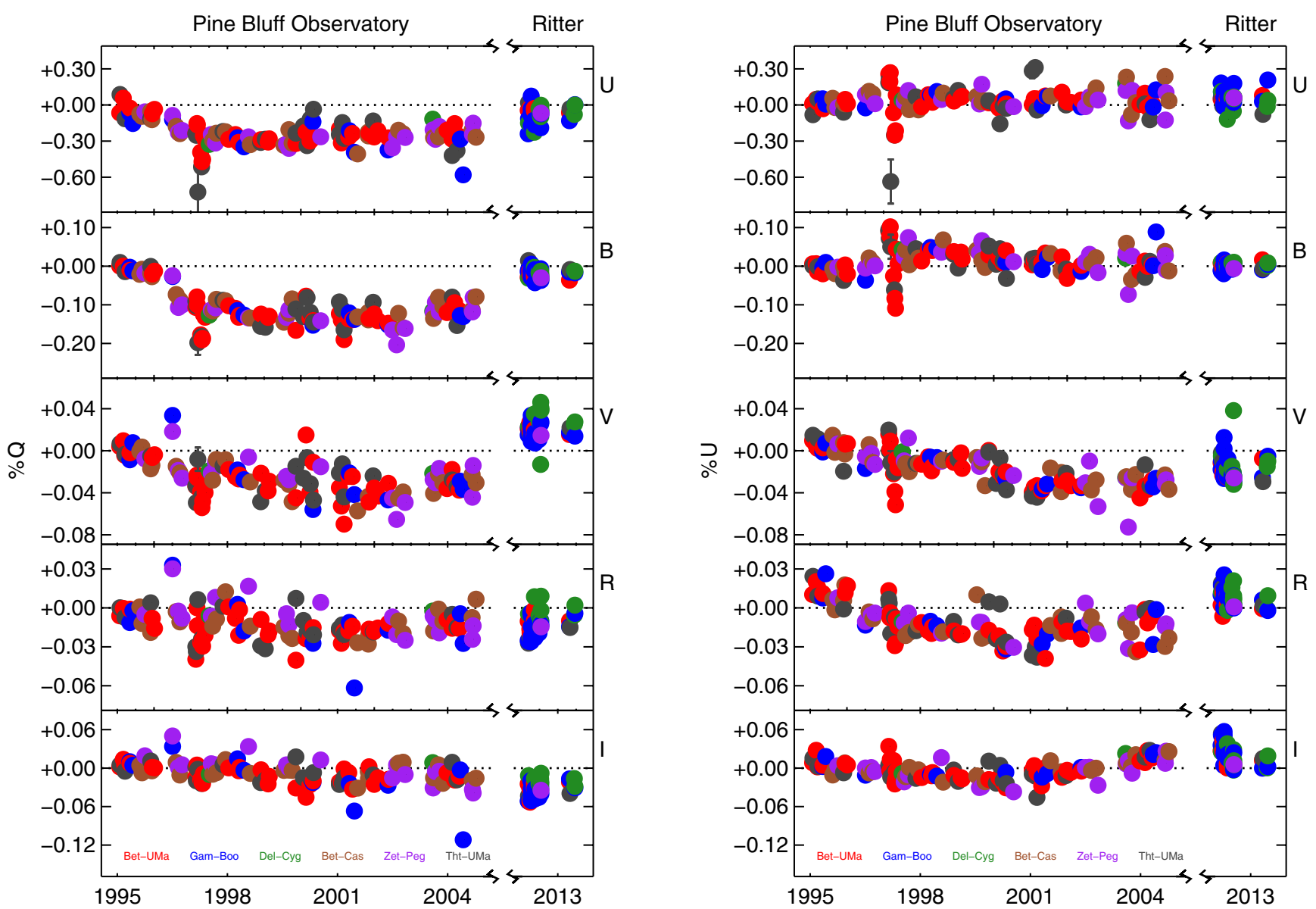

Fig. 7. (Color online) UBVRI synthetic filter polarizations in Stokes $Q$ (left) and Stokes $U$ (right) with no instrumental calibration applied for PBO and Ritter Observatory. $1-\sigma$ errors are plotted, though they are mostly smaller than the data points. Points are color coded based on the unpols, as shown in the legend at the bottom of each plot. There are individual plots for each filter, labeled on the right-hand side. Horizontal dotted lines show where $Q$ or $U$ are zero in each plot. Note the range and scale on the $y$-axis is different for each filter. The break in the $x$-axis denotes the roughly 7.5 year time period between the final observations at PBO and the new observations at Ritter Observatory.
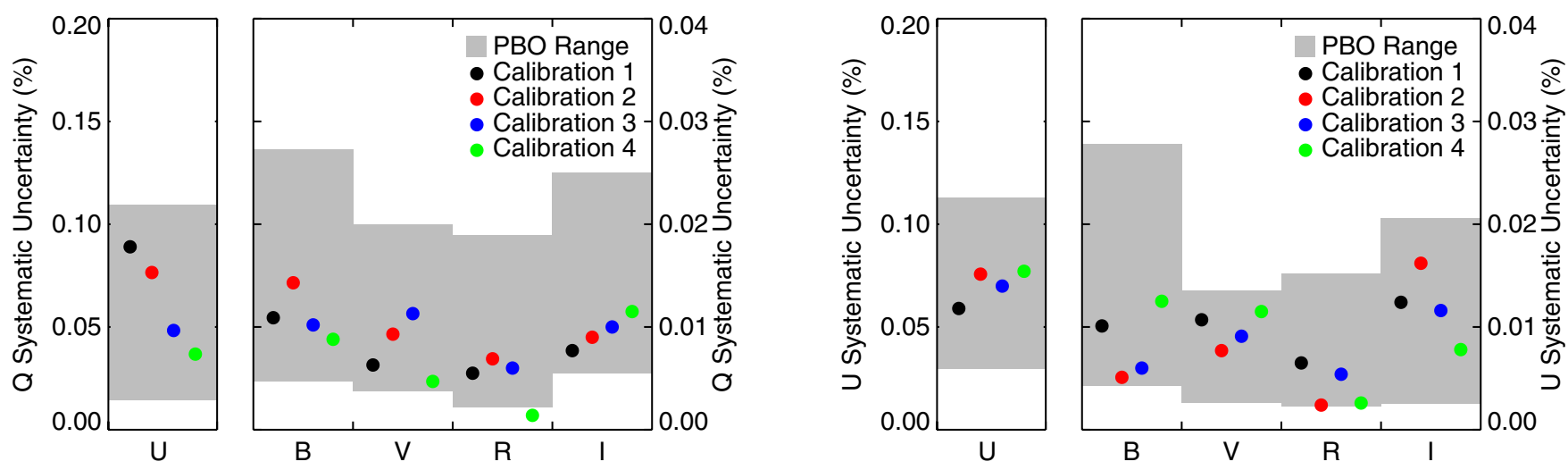

Fig. 8. (Color online) Comparison of PBO and Ritter Observatory systematic uncertainties in Stokes $Q$ (left) and Stokes $U$ (right). The gray regions show the range of UBVRI uncertainties for HPOL at PBO with the CCD detector (Table 5), while the filled circles show the uncertainties of each calibration for Ritter Observatory. Note the scales are different for the $U$ (left) and BVRI (right) synthetic filters. 


\section{Acknowledgments}

We acknowledge generous support from the Scott E. Smith Fund for Research at Ritter Observatory, and the University of Toledo Physics and Astronomy Department's Fund for Excellence. KSB acknowledges support from the American Astronomical Society in the form of a Small Research Grant. JLH acknowledges valuable support for the HPOL relocation project from the Mt. Cuba Astronomical Foundation and from the Fund for Astrophysical Research, Inc. in the form of a Theodore Dunham, Jr. Grant for Research in Astronomy. JLH has obtained funding for HPOL-related research from NSF awards AST-0807477 and AST-1210372, NASA ADAP award NNX13AF40G, and a Faculty Research Fund award from the University of Denver. In addition, JPW has obtained funding from NSF award AST-1412110, and KSB and JEB have obtained funding from NSF award AST1412135. JRL acknowledges support from the Sigma Xi Grants-in-Aid of Research Program. We wish to thank Rick Williams and Richard Bonomo from the University of Wisconsin-Madison for their technical expertise in getting HPOL restored to operation. We also thank Mike Brown from the University of Toledo for his help in installation and maintenance of HPOL at Ritter Observatory. We greatly appreciate the thoughtful and clarifying suggestions of Stefano Bagnulo and another anonymous referee. This research has made use of the SIMBAD database, operated at CDS, Strasbourg, France, and NASA's Astrophysics Data System Bibliographic Services.

\section{References}

Appenzeller, I. [1966] ZAp 64, 269.

Bailey, J., Lucas, P. W. \& Hough, J. H. [2010] MNRAS 405, 2570.

Bailey, J., Ulanowski, Z., Lucas, P. W. et al. [2008] MNRAS 386, 1016.
Behr, A. [1959] Veroeffentlichungen der UniversitaetsSternwarte zu Goettingen 7, 199.

Bessell, M. S. [1990] PASP 102, 1181.

Bjorkman, K. S., Meade, M. R., Nordsieck, K. H. et al. [1993] ApJ 412, 810.

Chandrasekhar, S. [1950] Radiative Transfer (Oxford University Press, London).

Clarke, D. [2010] Stellar Polarimetry (Wiley, Weinheim).

Davidson, J. W., Jr. [2013] "A new era of observational capability at Ritter Observatory: Spectropolarimetry from exoplanets to circumstellar disks and beyond," Ph.D. Dissertation, University of Toledo.

Gehrels, T. [1974] Planets, Stars and Nebulae Studied with Photopolarimetry (University of Arizona Press, Tuscon, Arizona).

Hough, J. H., Lucas, P. W., Bailey, J. A. et al. [2006] PASP 118, 1302.

Kemp, J. C., Henson, G. D., Steiner, C. T. \& Powell, E. R. [1987] Nature 326, 270.

Krautter, J. [1980] $A \& A S$ 39, 167.

Leroy, J. L. [1993] $A \& A S$ 101, 551.

Manset, N. \& Bastien, P. [1995] PASP 107, 483.

Nook, M. A. [1990] "The envelopes of RV Tauri variables," Ph.D. Dissertation, University of Wisconsin-Madison.

Nordsieck, K. H., Code, A. D., Anderson, C. M. et al. [1994] "Exploring ultraviolet astronomical polarimetry: Results from the Wisconsin Ultraviolet Photo-Polarimetry Experiment (WUPPE)," in Proc. SPIE, Vol. 2010, p. 2.

Nordsieck, K. H. \& Harris, W. [1996] "Future UV/visible spectropolarimetric studies of interstellar polarization at the University of Wisconsin," in Polarimetry of the Interstellar Medium, Special Issue, eds. Roberger, W. G. and Whittet, D. C. B., ASP Conf. Ser. 97, 100.

Piirola, V. [1977] A\&AS 30, 213.

Schröder, R. [1976] A\&AS 23, 125.

Serkowski, K. [1974] Planets, Stars, and Nebulae: Studied with Photopolarimetry, IAU Colloquium 23, p. 135.

Stokes, G. C. [1852] Trans. Cambridge Philos. Soc. 9, 399416.

Tinbergen, J. [1979] AधAS 35, 325.

Tinbergen, J. [1982] $A \mathscr{E} A$ 105, 53.

Tinbergen, J. [1996] Astronomical Polarimetry (Cambridge University Press, Cambridge, UK), p. 174.

Wolff, M. J., Nordsieck, K. H. \& Nook, M. A. [1996] AJ 111, 856. 